\title{
Comparative Study of Laparoscopic Assisted Vaginal Hysterectomy versus Vaginal Hysterectomy as Regards Blood Loss: Randomized Control Trial
}

Original Article

\author{
Sherif F. Hendawy, Mohamed E. Mohamed, Heba A. Allam, Elsayed A. Zaghalil
}

Department of Obstetrics and Gynecology, Faculty of Medicine, Ain-Shams University, Egypt

\begin{abstract}
Objective: A comparative research study conducted to evaluate and asses blood loss issues between laparoscopic assisted vaginal hysterectomy and vaginal hysterectomy.

Patients and Methods: A prospective randomized controlled research study trial to investigate blood loss and other surgical and clinical outcomes in comparison between VH and LAVH.

Results: No statistically significant difference was displayed between the research study groups concerning demographic parameters (age, BMI, parity uterine size) with $\mathrm{p}$ values $=0.224,0.845,0.296,0.175$ prospectively. No statistically significant difference between the research study groups concerning preoperative hemoglobin with $p$ value $=0.379$. However, postoperative $\mathrm{Hb}$ was statistically significantly greater within $\mathrm{VH}$ research group than within LAVH research group with $p$ value $=0.021$. Hemoglobin reduction was statistically significantly lower within VH than within LAVH with $p$ value $<0.001$.

Conclusion: our research group came to the conclusion that vaginal hysterectomy has a shorter operative time as well as less postoperative bleeding leading to lower $\mathrm{Hb}$ and $\mathrm{Hct}$ drop. Therefore it is better to conduct vaginal hysterectomy when surgically possible.
\end{abstract}

Key Words: blood loss, laparoscopic assisted vaginal hysterectomy, vaginal hysterectomy

Received: 12 November 2018, Accepted: 30 November 2018

Corresponding Author: Elsayed A. Zaghalil, MSc., Department of Obstetrics and Gynecology, Faculty of Medicine, AinShams University, Egypt, Tel.: 01064853063,E-mail: elsayedzaghalil@yahoo.com

ISSN: 2090-7265, May 2019, Vol.9, No. 2

\section{INTRODUCTION}

Vaginal hysterectomy is a surgery where the uterus is detached and extracted in professional surgical manner via the vagina. A vaginal surgical procedure for removal of uterus could be implemented if the uterus is not enlarged in a manner preventing the surgical possibility of the procedure ${ }^{[1]}$.

Uterine leiomyomas are one of the major indications for performing hysterectomy accounting for around $40 \%$ of all abdominal approach hysterectomies, various other indications involve endometriosis (12.8\%), malignant disease of the uterus (12.6\%), abnormal uterine bleeding (9.5\%), PID (3.7\%) and Prolapsed uterus (3.0\%). Interestingly uterine prolapse represents $44 \%$ of the indications of all vaginal hysterectomies. Gynecology surgeons are recently approaching non-descent type of vaginal hysterectomy being used for most benign indications and uteri of up to 12 weeks gestational volume could be removed safely from the vagina in an intact form.
However for moderately to large sized volumes of uteri with benign pathology, surgical techniques e.g wedge removal, uterine bisection, coring and morcellation could be implemented in intraoperative performance of the procedure in a trial to reduce uterine volume in a significant manner before surgical removal. On the other hand, large fibroids, PID, malignancy and most pathologically suspicious adnexal masses are. A number of complications could occur preferred to be approached abdominally by most surgeons $\mathbf{s}^{[2,3,4]}$. Various complications are possible to develop during performing the procedure such as bleeding, in cases that develop excessive amount of bleeding may need blood transfusion and/or reoperation to arrest the active bleeding. Additionally infection could occur however low-grade fever is a common clinical event after performing hysterectomy, however it is not always due to infection and with spontaneous resolution without implementing treatment. On the other hand, a high grade fever or persistent form of fever may denote development of an infectious complication. Serious infectious disorders develop in around less than $5 \%$ of cases and are usually managed sufficiently 
with intravenous form of antibiotics; rarely requires another interventional surgical procedure performance. Another common complication of hysterectomy is constipation usually managed with laxatives, dietary fibers. Narcotic pain control drugs usually increase the clinical possibility of developing constipation. Urinary retention is another possible unfavourable complication that occurs after vaginal hysterectomy that should be clinically diagnosed early and managed promptly by draining urine using a catheter till retention resolves, usually that occurs within 1 to 2 days ${ }^{[5,6,7]}$.

Any pelvic surgery raises the risk of blood clots formation and development in the large veins of lower limb or pulmonary vascular system. The risk is raised around six weeks after performing the surgical procedure. Surgical insult or damage to neighboring organs such as the urinary bladder, ureters and large and small bowel as they are anatomically located in the region of the lower abdomen and pelvis and surgical hazard of injury exists during performing hysterectomy. Bladder injury incidence is around $1-2 \%$ of cases undergoing vaginal hysterectomy, whereas bowel injury incidence is $<1 \%$ of cases. Surgical injury could usually be diagnosed intra operative and corrected within the operative procedure time of performance .Late diagnosis however may require another operative intervention ${ }^{[8,9,10]}$.

Early menopausal development may occur due to an interruption in normal blood perfusion to the ovarian tissue due to surgical removal of the uterus. Another form of hysterectomy named laparoscopic assisted vaginal hysterectomy is defined as surgical removal of the pelvic organs via the vagina but involves begining with ligation and cutting the ovarian anatomical attachments by usage and aid of laparoscopy implemented on the abdomen. LAVH is chosen for cases requiring vaginal hysterectomy but require reassurance that the ovaries are surgically removed, or cases that had prior surgical procedures making the vaginal route alone more surgically risky. However, there must be anatomical vaginal laxity and wideness. Surgical indications for performing LAVH involves the following:prior pelvic surgery, endometriosis ,prior cesarean section, suspicious adnexal pathology, uterine fibroid, ectopic pregnancy ${ }^{[11,12]}$.

\section{AIM OF THE WORK}

This research study compares and contrasts between laparoscopic assisted vaginal hysterectomy and vaginal hysterectomy as regards blood loss.

\section{PATIENTS AND METHODS}

This prospective randomized controlled research study trial, performed at Ain-Shams University Maternity Hospital, was conducted on 60 recruited cases after obtaining a written informed consent from all study subjects before participation. The research study cohort was randomized into two research groups; each study group involved 30 cases ; research group $\mathrm{V}$ who underwent vaginal hysterectomy and research group L who underwent laparoscopic assisted vaginal hysterectomy. Inclusive research criteria were as follows age $>40$ years, uterine size $<12$ weeks, benign uterine pathology, BMI $<25$, multiparous women and vaginal prolapse. Exclusive research criteria were as follows age $<40$ years, uterine size $>16$ weeks, malignant uterine pathology, intra-abdominal adhesions e.g due to laparotomy and previous uterine scar, nulliparous, contraindication of laparoscopy (eg. severe cardiac and chest diseases). All cases were subjected to preoperative evaluation involving detailed clinical history taking, physical examination, general examination involving the whole body, body mass index, local examination i.e abdominal examination, pelvic examination including evaluation of sub-pubic angle, uterine size, mobility and descent, presence of any adnexal mass and tenderness and depth of posterior vaginal fornix, preoperative investigation, a preoperative trans-vaginal sonography to evaluate the uterine volume, gross uterine pathology and any adnexal pathology. The preoperative laboratory workup was performed including hemoglobin concentration and hematocrit value and full lab chemistry.

The bleeding assessment protocols used were: the first protocol used is the visual estimation method in which after skin closure the anesthetist, the gynecologist as well as the scrub nurse will be asked to estimate according to what they notice during surgery how much blood the patient lost in $\mathrm{ml}$. The recorded values will be kept with the circulating nurse. The second protocol used was the mathematical calculation in which the intraoperative blood loss has been estimated and evaluated by obtaining the hematocrit (Hct) indices directly after hospital admission and one hour in postoperative period within recovery ward.

\section{$\underline{E B V x}(\mathbf{H i}-\mathbf{H f})$ $\underline{\mathrm{Hi}}=\mathrm{ABL}$}

$$
\begin{aligned}
& \mathrm{H}_{\mathrm{i}}=\text { initial Hct } \\
& \mathrm{H}_{\mathrm{f}}=\text { final lowest acceptable Hct }
\end{aligned}
$$

ABL: Allowable Blood Loss

$$
\mathrm{EBV}=\text { weight }(\mathrm{kg}) \mathrm{x} \text { average blood volume }
$$

EBV: Estimated Blood Volume 
The third protocol used measurement of blood in the suction bottle and the fourth protocol used was displayed in Table 1

\begin{tabular}{ll}
\hline & Estimated blood \\
\hline Gauze & $30 \mathrm{cc}$ \\
Small soaked swab & $60 \mathrm{cc}$ \\
Large soaked swab & $350 \mathrm{cc}$ \\
Full kidney dish & $500 \mathrm{cc}$ \\
\hline
\end{tabular}

Primary research outcome measurements were as follows: estimated blood loss and need for blood transfusion, operative time, intra-operative complications, and requirement for laparotomy due to whichever surgical difficulty or slipped surgical pedicle, intestinal or urinary tract surgical insult.

Secondary research outcome measurements were as follows: postoperative complications, postoperative pain evaluation using the visual analogue scale and requirement for analgesia, postoperative hospital stay and wound infection.

\section{STATISTICAL ANALYSIS}

Descriptive statistics for obtained research variables are displayed and expressed as range, mean and standard deviation (for metric data); range, median and interquartile range (for discrete data); and number and proportions (for categorical data). SPSS program Version 15.0 will be used for data presentation and statistical analysis.

\section{RESULTS}

The investigated groups were described as follows:

Group VH: 30 women who had vaginal hysterectomy. Group LAVH: 30 women who had laparoscopic assisted vaginal hysterectomy.

Table 1: Demographic characteristics among the studied groups

\begin{tabular}{ccccc}
\hline Items & Measure & $\begin{array}{c}\mathrm{VH} \\
(\mathrm{N}=30)\end{array}$ & $\begin{array}{c}\text { LAVH } \\
(\mathrm{N}=30)\end{array}$ & P-value $^{\wedge}$ \\
\hline $\begin{array}{c}\text { Age } \\
\text { (years })\end{array}$ & Mean \pm SD & $46.0 \pm 2.8$ & $45.2 \pm 2.7$ & 0.224 \\
Range & $42.0-54.0$ & $40.0-53.0$ & \\
$\begin{array}{c}\mathrm{BMI} \\
(\mathrm{kg} / \mathrm{m} 2)\end{array}$ & Mean $\pm \mathrm{SD}$ & $25.5 \pm 1.7$ & $25.6 \pm 1.8$ & 0.845 \\
& Range & $22.3-28.9$ & $22.6-30.4$ & \\
Parity & Mean $\pm \mathrm{SD}$ & $3.0 \pm 1.0$ & $3.3 \pm 1.0$ & 0.296 \\
& Range & $1.0-5.0$ & $1.0-5.0$ & \\
$\begin{array}{c}\text { Uterine } \\
\text { size } \\
(\text { weeks })\end{array}$ & Mean $\pm \mathrm{SD}$ & $8.9 \pm 1.4$ & $8.4 \pm 1.4$ & \\
& Range & $7.0-12.0$ & $7.0-12.0$ & 0.175 \\
& & & &
\end{tabular}

$\wedge$ Independent t-test
No statistically significant difference between the research study groups as regards demographic variables (age, BMI, parity uterine size) with $p$ values $=0.224,0.845,0.296,0.175$ consecutively.

Table 2: Blood loss $(\mathrm{mL})$ among the studied groups

\begin{tabular}{|c|c|c|c|}
\hline & $\begin{array}{c}\text { VH } \\
(\mathrm{N}=30)\end{array}$ & $\begin{array}{l}\text { LAVH } \\
(\mathrm{N}=30)\end{array}$ & P-value ${ }^{\wedge}$ \\
\hline $\begin{array}{l}\text { Mean } \pm \text { SD } \\
(\mathrm{ml})\end{array}$ & $126.7 \pm 48.6$ & $279.7 \pm 113.8$ & \multirow[t]{2}{*}{$<0.001 *$} \\
\hline Range (ml) & $37.2-247.7$ & $91.4-448.5$ & \\
\hline \multicolumn{4}{|c|}{ Value of VH over LAVH } \\
\hline Items & Mean \pm SD & \multicolumn{2}{|c|}{$95 \% \mathrm{CI}$} \\
\hline $\begin{array}{l}\text { Blood loss } \\
\text { reduction }(\mathrm{ml})\end{array}$ & $153.0 \pm 22.6$ & \multicolumn{2}{|c|}{$107.8-198.3$} \\
\hline
\end{tabular}

Independent t-test, CI: Confidence interval, *Significant

Blood loss was statistically significantly lower in $\mathrm{VH}$ research group than LAVH research group with $p$ value $<0.001$.

Table 3 and Fig. 1 show that blood loss was significantly lower among VH than among LAVH.

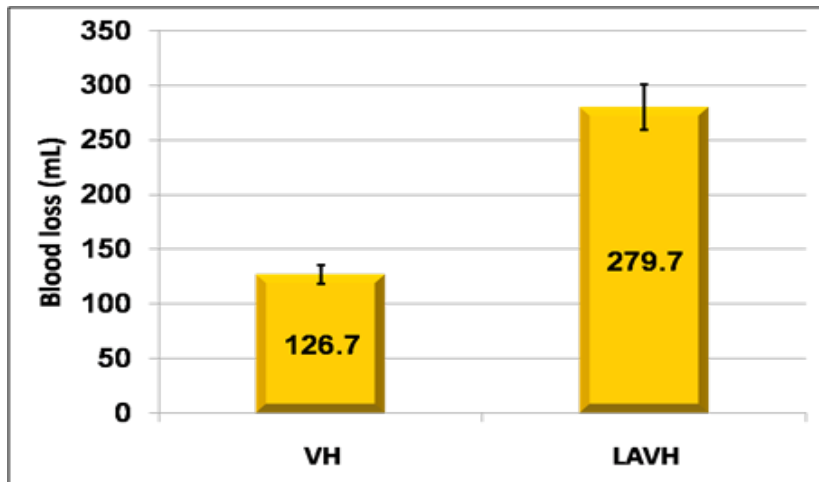

Fig. 1: Blood loss among the studied groups

Table 3: Hemoglobin (gm/dL) among the studied groups

\begin{tabular}{|c|c|c|c|c|}
\hline Time & Measure & $\begin{array}{c}\mathrm{VH} \\
(\mathrm{N}=30)\end{array}$ & $\begin{array}{l}\text { LAVH } \\
(\mathrm{N}=30)\end{array}$ & P-value ${ }^{\wedge}$ \\
\hline \multirow{2}{*}{$\begin{array}{c}\text { Pre } \\
\text { Operative } \\
\mathrm{Hb}(\mathrm{g} / \mathrm{dl})\end{array}$} & Mean \pm SD & $12.3 \pm 1.0$ & $12.1 \pm 0.7$ & \multirow[b]{2}{*}{0.379} \\
\hline & Range & $11.0-14.7$ & $10.8-13.7$ & \\
\hline \multirow{2}{*}{$\begin{array}{c}\text { Post } \\
\text { Operative } \\
\mathrm{Hb}(\mathrm{g} / \mathrm{dl})\end{array}$} & Mean \pm SD & $12.1 \pm 1.0$ & $11.5 \pm 0.7$ & \multirow[t]{2}{*}{$0.021 *$} \\
\hline & Range & $10.8-14.5$ & $10.3-13.1$ & \\
\hline \multirow{2}{*}{$\begin{array}{l}\text { Reduction } \\
\mathrm{Hb}(\mathrm{g} / \mathrm{dl})\end{array}$} & Mean \pm SD & $0.3 \pm 0.1$ & & \multirow{2}{*}{$<0.001 *$} \\
\hline & Range & $0.1-0.5$ & $0.2-1.0$ & \\
\hline \multicolumn{5}{|c|}{ Value of VH over LAVH } \\
\hline Items & \multicolumn{2}{|c|}{ Mean \pm SD } & \multicolumn{2}{|c|}{$95 \% \mathrm{CI}$} \\
\hline $\begin{array}{l}\text { Reduction } \\
\text { difference }\end{array}$ & \multicolumn{2}{|c|}{$0.3 \pm 0.1$} & \multicolumn{2}{|c|}{$0.2-0.4$} \\
\hline
\end{tabular}

Independent t-test, CI: Confidence interval, *Significant 
Table 3 and Fig. 2 display that no statistically significant difference between the research study groups regarding preoperative hemoglobin with $p$ value $=0.379$. Postoperative hemoglobin was statistically significantly greater within VH research group than within LAVH research group with $p$ value $=0.021$. Hemoglobin reduction was statistically significantly lower within $\mathrm{VH}$ than within LAVH with $p$ value $<0.001$.

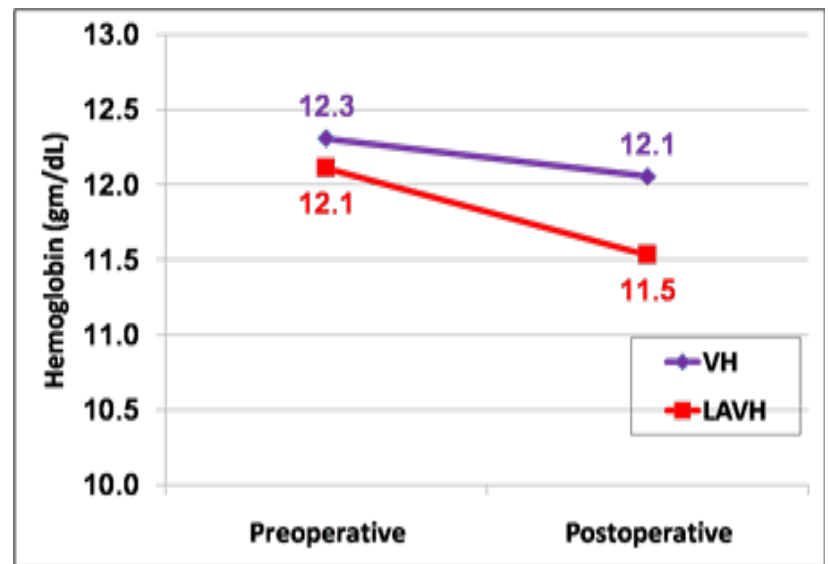

Fig. 2: Hemoglobin among the studied groups

Table 4: Hematocrit (\%) among the studied groups

\begin{tabular}{|c|c|c|c|c|}
\hline Time & Measure & $\begin{array}{c}\mathrm{VH} \\
(\mathrm{N}=30)\end{array}$ & $\begin{array}{l}\text { LAVH } \\
(\mathrm{N}=30)\end{array}$ & P-value ${ }^{\wedge}$ \\
\hline \multirow{2}{*}{$\begin{array}{l}\text { Pre } \\
\text { operative }\end{array}$} & Mean \pm SD & $36.3 \pm 3.0$ & $35.9 \pm 2.3$ & \multirow{2}{*}{0.576} \\
\hline & Range & $32.0-43.9$ & $31.5-40.5$ & \\
\hline \multirow{2}{*}{$\begin{array}{l}\text { Post } \\
\text { operative }\end{array}$} & Mean \pm SD & $35.6 \pm 3.0$ & $34.2 \pm 2.4$ & \multirow{2}{*}{$0.049^{*}$} \\
\hline & Range & $31.3-43.2$ & $29.1-38.8$ & \\
\hline \multirow{2}{*}{ Reduction } & Mean \pm SD & $0.8 \pm 0.3$ & $1.8 \pm 0.7$ & \multirow{2}{*}{$<0.001^{*}$} \\
\hline & Range & $0.2-1.5$ & $0.6-3.0$ & \\
\hline \multicolumn{5}{|c|}{ Value of VH over LAVH } \\
\hline Items & \multicolumn{2}{|c|}{ Mean \pm SD } & \multicolumn{2}{|c|}{$95 \% \mathrm{CI}$} \\
\hline $\begin{array}{l}\text { Reduction } \\
\text { difference }\end{array}$ & \multicolumn{2}{|c|}{$1.0 \pm 0.1$} & \multicolumn{2}{|c|}{$0.7-1.3$} \\
\hline
\end{tabular}

Independent t-test, CI: Confidence interval, *Significant

Table 4 and Fig. 3 show that no statistically significant difference between thestudied groups regarding preoperative hematocrit $p$ value $=0.576$. Postoperative hematocrit was statistically significantly higher among VH group than among LAVH group with $p$ value $=0.049$. Hematocrit reduction was significantly lower among $\mathrm{VH}$ than among LAVH $p$ value $<0.001$.

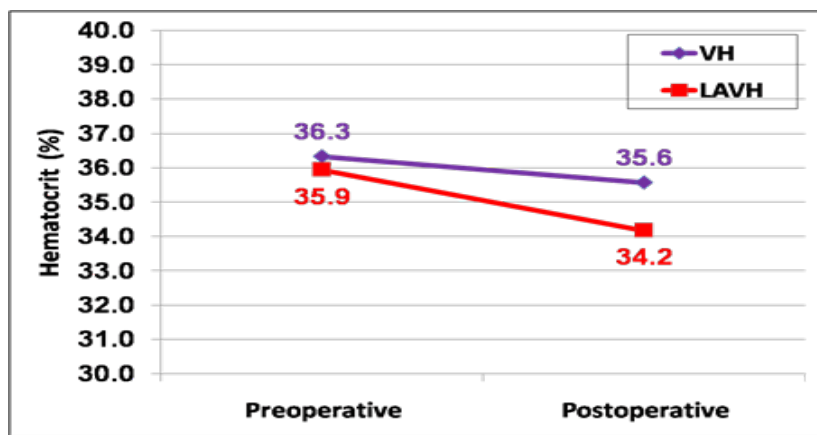

Fig. 3: Hematocrit among the studied groups

Table 5: Operative duration and complications among the studied groups

\begin{tabular}{lccc}
\hline Variables & $\begin{array}{c}\text { VH } \\
(\mathrm{N}=30)\end{array}$ & $\begin{array}{c}\text { LAVH } \\
(\mathrm{N}=30)\end{array}$ & P-value \\
\hline Laparotomy & $0(0.0 \%)$ & $0(0.0 \%)$ & $\# 1.000$ \\
$\begin{array}{l}\text { Hemorrhage } \\
\begin{array}{l}\text { Urinary tract } \\
\text { injury }\end{array}\end{array}$ & $0(0.0 \%)$ & $1(3.3 \%)$ & $\# 1.000$ \\
& $1(3.3 \%)$ & $2(6.7 \%)$ & $\# 1.000$
\end{tabular}

\begin{tabular}{|c|c|c|c|}
\hline \multirow{2}{*}{$\begin{array}{l}\text { Operative } \\
\text { duration } \\
\text { (minutes) }\end{array}$} & Mean \pm SD & $88.8 \pm 19.6$ & $119.2 \pm 15.3$ \\
\hline & Range & $59.0-124.0$ & $90.0-141.0$ \\
\hline
\end{tabular}

Value of VH over LABH

\begin{tabular}{lll} 
Items & Mean $\pm \mathrm{SE}$ & $95 \% \mathrm{CI}$ \\
$\begin{array}{l}\text { Duration } \\
\text { reduction }\end{array}$ & $30.5 \pm 4.5$ & $21.4-39.6$ \\
\hline $\begin{array}{l}\text { \#Fisher's Exact } \\
\text { interval, *Significant }\end{array}$ & Independent-test, & CI: Confidence
\end{tabular}

Table 5 and Fig. 4 show that no significant difference between the studied groups regarding complications with $\mathrm{p}$ value in all aspects $=1.00$. Operative duration was significantly shorter among $\mathrm{VH}$ than among LAVH with $p$ value $<0.001$.

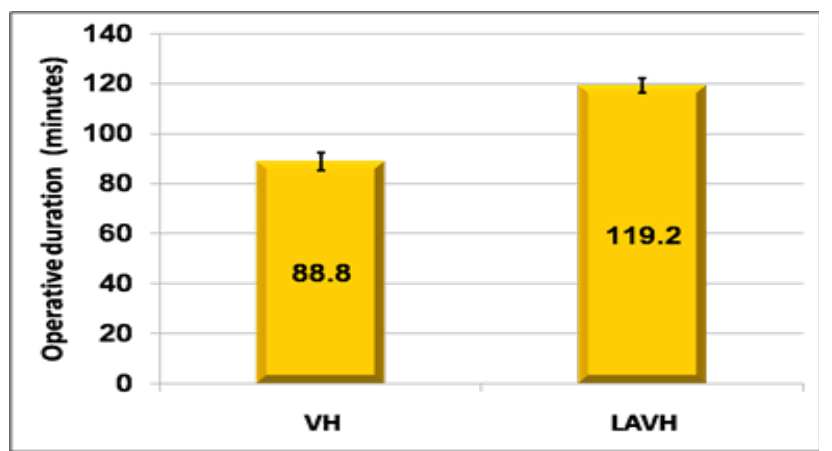

Fig. 4: Operative duration among the studied groups 
Table 6: Hospital stay and postoperative complications among the studied groups

\begin{tabular}{|c|c|c|c|c|}
\hline \multicolumn{2}{|l|}{ Variables } & $\begin{array}{c}\mathrm{VH} \\
(\mathrm{N}=30)\end{array}$ & $\begin{array}{l}\text { LAVH } \\
(\mathrm{N}=30)\end{array}$ & P-value \\
\hline \multicolumn{2}{|c|}{ Wound infection } & $0(0.0 \%)$ & $1(3.3 \%)$ & $\# 1.000$ \\
\hline \multicolumn{2}{|c|}{ Pyrexia $>38.0^{\circ} \mathrm{C}$} & $1(3.3 \%)$ & $1(3.3 \%)$ & $\# 1.000$ \\
\hline \multirow{2}{*}{$\begin{array}{l}\text { Stay } \\
\text { (days) }\end{array}$} & Mean \pm SD & $2.7 \pm 0.7$ & $3.1 \pm 0.3$ & \multirow{2}{*}{${ }^{\wedge} 0.005^{*}$} \\
\hline & Range & $1.0-3.0$ & $3.0-4.0$ & \\
\hline Items & \multicolumn{2}{|c|}{ Mean \pm SE } & \multicolumn{2}{|c|}{$95 \% \mathrm{CI}$} \\
\hline $\begin{array}{l}\text { Stay } \\
\text { reduction }\end{array}$ & \multicolumn{2}{|c|}{$0.4 \pm 0.1$} & \multicolumn{2}{|c|}{$0.1-0.7$} \\
\hline
\end{tabular}

${ }^{\wedge}$ Independent t-test, \#Fisher's Exact test, CI: Confidence interval, *Significant

Table 6 and Fig. 5 show that no statistically significant difference between the studied groups regarding postoperative complications with $p$ value $=1.00$. Hospital stay was statistically significantly shorter among VH than among LAVH with $p$ value $<0.005$.

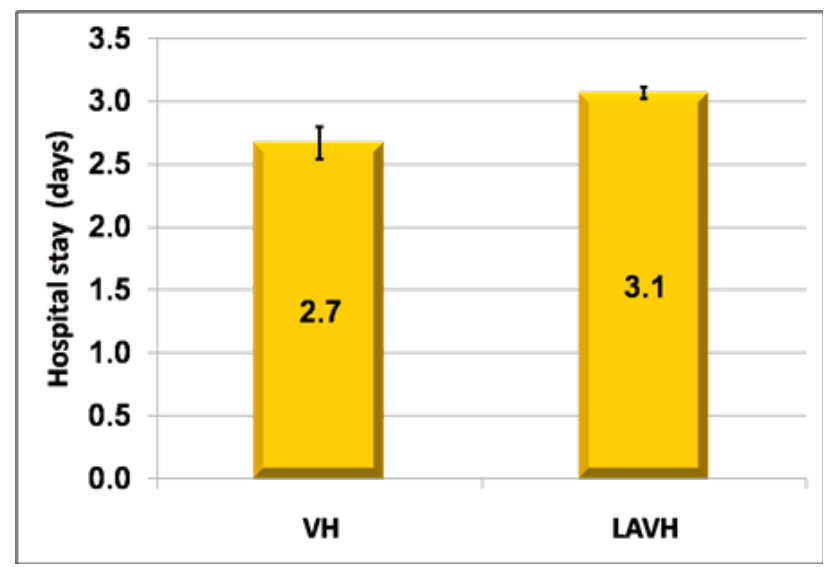

Fig. 5: Hospital stay among the studied groups

\section{DISCUSSION}

Recently, hysterectomy is one of the most frequent surgical operations performed. It was displayed that $1 / 3$ of the females have undergone hysterectomy by age 60 , with 600000 annually performed operations in USA ${ }^{[1,2,3]}$. Previously hysterectomy was performed by the classic abdominal approach that is still extensively performed currently. Even though it is considered one of the safest procedures it causes a disfiguring scar due to large laparotomy surgical skin incision .Minimally invasive surgical protocols have been raised at the present time consecutively to handle the negative aspect of classic abdominal hysterectomy such as vaginal hysterectomy and laparoscopic assisted vaginal hysterectomy ${ }^{[5,6,7]}$. Various research studies had displayed and revealed that vaginal hysterectomy have a superior post-operative clinical outcomes in comparison to laparoscopic assisted vaginal hysterectomy that is a good reason to be considered a priority for begin gynecological pathologies. On the other hand, various research studies displayed that that both vaginal hysterectomy and laparoscopic assisted vaginal hysterectomy are considered to have the same post-operative surgical and clinical outcomes with no advantage of one surgical approach vs. the other concerning the post-operative complications. In surgical case scenarios in which vaginal hysterectomy is difficult to implement safely such as cases with large bulky uterus that is considered complex to excise via the vaginal opening ${ }^{[8,910,15,16,17]}$.

In addition to cases having narrow vaginal opening like nulliparous and patients with severe grades of endometriosis LAVH is a safe alternative. In Egypt, according to our knowledge, no research studies have been performed to compare the post-operative clinical and surgical outcome of vaginal hysterectomy versus laparoscopic assisted vaginal hysterectomy. A Prospective randomized controlled research study have been performed in Ain-Shams University Maternity Hospital on cases that undergo hysterectomy for begin pathologies. Seventy two were evaluated for eligibility. Eleven of them didn't meet the research study inclusive criteria and one case refused participation. As a result, the research study was finally performed on 60 cases. The study subjects were categorized in two equal research groups. Research group $\mathrm{V}$ had undergone vaginal hysterectomy and research group $\mathrm{L}$ had undergo laparoscopic assisted vaginal hysterectomy. Both groups have been evaluated for post-operative blood loss, intra-operative and post-operative issues. The two research groups have been matched as regards age, BMI, parity and uterine volume .This current research study displayed that post-operative blood loss, $\mathrm{Hb}$ and Hct drop were statistically significantly lower within the research group that have undergone vaginal hysterectomy in comparison and contrast the other research group study subjects that have undergone laparoscopic assisted vaginal hysterectomy $p$ value $<0.001$.

Additionally, the current research study have displayed no statistically significant difference between both research groups concerning preoperative $\mathrm{Hb}$ and Hct with $p$ value $=0.576$ whereas postoperative $\mathrm{Hb}$ and $\mathrm{Hct}$ was statistically significantly greater within vaginal hysterectomy research group more than within LAVH research group. As regards the intra operative complications of hysterectomy in the 
form of hemorrhage, injury to the urinary tract and laparotomy scar in the current research study revealed no statistically significant intra operative complications between both investigated types of hysterectomy with $\mathrm{p}$ value in all aspects $=1.00$ (laparotomy, haemorrhage, urinary tract injury).

H. Eggemann et al. research group priory performed a research with the aim compare the effect of closing the peritoneum on postoperative pain after vaginal hysterectomy and laparoscopic-assisted vaginal hysterectomy that was a prospective, randomized, double-blind research study to evaluate and asses primary clinical outcome such as the postoperative pain after $\mathrm{VH}$ and $\mathrm{LAVH}$ with and without closing the peritoneum. Evaluation of postoperative pain was assessed and evaluated by using visual analogue scale. Study subjects were recruited between August, 2007 and July, 2014. A cohort of 192 cases with benign uterine pathology was categorized in 4 groups: LAVH and VH with and without peritoneal closure, consecutively. The study subject's features involving parity, BMI, prior abdominal operations, and uterine weight were matched between the research groups. The cases that undergone LAVH were statistically significantly younger in age ( $p$ value $=0.0443$ ). LAVH was correlated with raised postoperative pain and decreased patients' ambulation in the first 72 and $24 \mathrm{~h}$, consecutively, after performing surgery. The usage of analgesia stayed similar in all research categorical groups. The operative time was statistically significantly shorter after vaginal hysterectomy $(\mathrm{VH}+\mathrm{PC} 59 \pm 17 ; \mathrm{VH}-\mathrm{PC} 56 \pm 19)$ than after laparoscopic assisted vaginal hysterectomy $(\mathrm{LAVH}+\mathrm{PC} 106 \pm 29 \mathrm{~min} ; \mathrm{LAVH}-\mathrm{PC} 99 \pm 30)$ $(p<0.0001)$. The process of peritoneal closure did not influence the cases clinical outcome. The amount blood loss, the drop in $\mathrm{Hb}$, the hospitalization period, and the frequency of intra- and postoperative complications were statistically similar in all research group categories. The research group concluded that vaginal hysterectomy is correlated with shorter operative time and decreased postoperative pain in comparison to laparoscopic assisted vaginal hysterectomy. This research study shows similarity and harmony with our research study as regards operative time but our research did not asses the pain as a separate aspect ${ }^{[14]}$.

However, Elisabeth Gottschalk, et al. research group performed a research study about total laparoscopic radical hysterectomy which is correlated with higher risk for urologic complications. It comprised mainly of three operative steps; meticulous laparoscopic staging, surgical performance creation of a tumor modified vaginal cuff, and laparoscopic surgical transaction of parametrium on both sides. In a retrospective manner, the research group assessed the findings and data of 122 cases undergoing total laparoscopic radical hysterectomy for early stage cervical cancer $(n=110)$ or stage II endometrial cancer $(n=12)$ between January 2007 and December 2009 at Charite' University Hospital, Berlin Germany. All cases recruited for the research study undergone total laparoscopic radical hysterectomy without conversion to laparotomy. Mean operative time was $300 \mathrm{~min}$ and mean loss of blood was $123 \mathrm{cc}$. Commonly around 36 lymph nodes per case were obtained. Intra- and postoperative complication rates were $0 \%$ and $13.1 \%$, consecutively. Resection margin have been in sound in all cases. After a median period of clinical follow-up for around 19 months, survival disease-free rate and overall survival rate for all 110 cancer cervix cases was $94 \%$ and $98 \%$ consecutively The research group concluded that total laparoscopic radical hysterectomy is an applicable substitute to abdominal route radical hysterectomy and total laparoscopic radical hysterectomy in cases with early stage cancer cervix and cancer endometrium stage II with negligible intra operative complications and identical clinical oncologic outcomes. This research study differed from ours in investigating out comes in malignant causes for radical hysterectomy and differed in that surgical approach was totally laparoscopic ${ }^{[15]}$.

\section{CONCLUSION AND RECOMMENDATION}

Currently there's a raised prevalence of hysterectomy attributed to the raised in the frequency of diagnosing of uterine benign pathologies e.g. fibroids and endometriosis. The commonly performed hysterectomy approach is the traditional abdominal one which leaves a large laparotomy scar. Nowadays, new hysterectomy approaches such as LAVH and VH were developed aiming to manage this problem. Up till now there is a debate about which of these two approaches is better regarding post-operative complications especially post-operative bleeding. A prospective randomized controlled study was done in Ain-Shams University Maternity Hospital on sixty patients that undergone hysterectomy for begin conditions. They were divided in two equal groups; one had undergone $\mathrm{VH}$ and the other had undergone LAVH. The two groups were matched according to age, BMI, parity and uterine size. Both were assessed for post-operative blood loss, intra-operative and post-operative problems. This study revealed a significant low post-operative Blood loss, hemoglobin and hematocrit reduction in those how performed $\mathrm{VH}$ as well as a significant high postoperative hemoglobin and hematocrit. It revealed also a significantly shorter operative duration as well as hospital stay among those who undergo VH. Finally, 
this study showed no significant intraoperative or postoperative complications between these new types of hysterectomy approaches.

From this research, we concluded that vaginal hysterectomy have a shorter operative time in addition to less post-operative bleeding causing a lower $\mathrm{Hb}$ and Hct fall which causes in conclusion a lower hospital stay. Therefore, it is better to perform $\mathrm{VH}$ when ever surgically possible.

\section{CONFLICT OF INTEREST}

There are no conflicts of interest.

\section{REFERENCES}

1. Whiteman MK, Hillis SD, Jamieson DJ, Morrow B, Podgornik MN,Brett KM, et al. Inpatient hysterectomy surveillance in the UnitedStates, 20002004-. Am J Obstet Gynecol. 2008;198:34.e134-.e7

2. Clarke-Pearson DL, Geller EJ. (2013): Complications of hysterectomy. Obstet Gynecol 121: 654-673.

3. Akyol D, Esinler I, Guven S, Salman MC, Ayhan A. Vaginal hysterectomy: results and complications of 886 patients. J Obstet Gynaecol.2006;26:777-781.

4. Yi YX, Zhang W, Zhou Q, Guo WR, Su Y. Laparoscopic-assisted vaginal hysterectomy vs abdominal hysterectomy for benign disease: a meta-analysis of randomized controlled trials. Eur JObstet Gynecol Reprod Biol.2011;159:1-18.

5. Geller EJ. (2014): Vaginal hysterectomy: the original minimally invasive surgery. Minerva Ginecol 66: 23-33.

6. Nieboer TE, Hendriks JC, Bongers MY, Vierhout ME, Kluivers KB. (2012): Quality of life after laparoscopic and abdominal hysterectomy: a randomized controlled trial. Obstet Gynecol 119: 85-91.

7. Chen B, Ren DP, Li JX, Li CD. (2014): Comparison of vaginal and abdominal hysterectomy:A prospective non-randomized trial. Pak J Med Sci 30: 875-879.

8. Johnson N, Barlow D, Lethaby A, Tavender E, Curr E, Garry R. Surgical approach to hysterectomy for benign gynaecologicaldisease. Cochrane Database Syst Rev 2006;(2): CD003677.
9. Hwang JL, Seow KM, Tsai YL, Huang LW, Hsieh BC, Lee C. Comparative study of vaginal, laparoscopically assisted vaginal and abdominal hysterectomies for uterine myoma larger then $6 \mathrm{~cm}$ in diameter or uterus weighting at least $450 \mathrm{~g}$ : a prospective randomized study. Acta Obstet Gynecol Scand. 2002;81:1132-1138.

10. Francesco Sesti, MD, Velia Ruggeri, MD, Adalgisa Pietropolli, MD, Emilio Piccione, MD Laparoscopically Assisted Vaginal Hysterectomy Versus Vaginal Hysterectomy for Enlarged Uterus Journal of the Society of Laparoendoscopic Surgeons. (2008)12:246-251

11. Ribeiro SC, Ribeiro RM, Santos NC, Pinotti JA. A randomized Laparoscopically Assisted Vaginal Hysterectomy vs Vaginal Hysterectomy for Enlarged Uterus, (2008)12:246-251

12. Cook JR, O'Shea RT, Seman EI. Laparovaginal hysterectomy:a decade of evolution. Aust N J Obstet Gynaecol. 2004;44:111-116.

13. Ibeanu OA, Chesson RR, Echols KT, Nieves M, Busangu F, Nolan TE. Urinary tract injury during hysterectomy based on universal cystoscopy. Obstet Gynecol. 2009;113(1):610-. doi: 10.1097/ AOG.0b013e31818f6219.

14. H. Eggemann, A. Ignatov ,H. Frauchiger-Heuer T. Amse S. D. Costa Laparoscopic-assisted vaginal hysterectomy versus vaginal hysterectomy for benign uterine diseases: a prospective, randomized, multicenter, double-blind trial (LAVA) Archives of Gynecology and Obstetrics 2018 https:/doi. org/10.1007/s00404-017-4647-7

15. Elisabeth Gottschalk, Malgorzata Lanowska, Vito Chiantera, Simone Marnitz, Achim Schneider, Verena Brink-Spalink, Kati Hasenbein, Christhardt Koehler, Vaginal-Assisted Laparoscopic Radical Hysterectomy: Rationale, Technique, Results JSLS (2011)15:451-459

16. Aarts JW, Nieboer TE, Johnson N et al (2015) Surgical approach to hysterectomy for benign gynaecological disease. Cochrane Database Syst Rev (8):CD003677. http s://doi.org/10.1002 /1465 1858 .CD00 3677 .pub5

17. Guo Y, Tian X, Wang L (2013) Laparoscopically assisted vaginal hysterectomy vs vaginal hysterectomy: meta analysis. J Minim Invasive Gynecol 20:15-21. 